\title{
POSITIVE SCALAR CURVATURE AND CALLIAS-TYPE INDEX THEOREMS FOR PROPER ACTIONS
}

\author{
HAO GUO
}

(Received 20 October 2018; first published online 7 January 2019)

2010 Mathematics subject classification: primary 58B34; secondary 19K35, 19K56, 19L47, 47C15, $58 \mathrm{~J} 20$.

Keywords and phrases: positive scalar curvature, equivariant index, Callias operator.

This thesis by publication is a study of the equivariant index theory of Dirac operators and Callias-type operators in two distinct settings, namely on cocompact and noncocompact manifolds with a Lie group action.

The first two chapters are a short resumé of Dirac operators and index theory and form a common introduction to the papers in the appendices.

The first appendix [2] is joint work with my supervisors, Elder Professor Mathai Varghese and Dr Hang Wang. For $G$ an almost-connected Lie group acting properly and cocompactly on a manifold $M$, we study $G$-index theory of $G$-invariant Dirac operators. By establishing Poincaré duality for equivariant $K$-theory and $K$-homology, we are able to extend the scope of our results to include all elements of equivariant analytic $K$-homology, which we also show is isomorphic to equivariant geometric $K$-homology. Our results are applied to prove: a rigidity result for almost-complex manifolds, generalising a vanishing theorem of Hattori; an analogue of Petrie's conjecture; and Lichnerowicz-type obstructions to $G$-invariant Riemannian metrics on $M$.

The second appendix [1] studies the much more general situation where the quotient $M / G$ is noncompact and $G$ is an arbitrary Lie group. We define G-Callias-type operators and show that they are $C^{*}(G)$-Fredholm by adapting analysis by Kasparov to new Hilbert $C^{*}(G)$-module analogues of Sobolev spaces. Questions of adjointability, regularity and essential self-adjointness are addressed in detail. The estimates on $G$ Callias-type operators are based on the work of Bunke in the nonequivariant context. We construct explicit admissible endomorphisms for $G$-Callias-type operators from the $K$-theory of the Higson $G$-corona of $M$, a highly nontrivial group. The index

Thesis submitted to the University of Adelaide in July 2018; degree approved 8 August 2018; supervisors Mathai Varghese and Hang Wang.

(C) 2019 Australian Mathematical Publishing Association Inc. 
theory developed here is applied to prove a general obstruction theorem for $G$-invariant metrics of positive scalar curvature in the noncocompact setting.

\section{References}

[1] H. Guo, 'Index of equivariant Callias-type operators and invariant metrics of positive scalar curvature'. Preprint, 2018, arXiv:1803.05558.

[2] H. Guo, V. Mathai and H. Wang, 'Positive scalar curvature and Poincaré duality for proper actions'. J. Noncommut. Geom., to appear. Preprint, 2016, arXiv:1609.01404.

HAO GUO, School of Mathematical Sciences, University of Adelaide, Adelaide, South Australia 5005, Australia

e-mail: oug.oah@gmail.com 